\title{
Die Zukunft des Fachbereichs „Künstliche Intelligenz“
}

\author{
Antonio Krüger
}

Online publiziert: 8 . November 2011

(C) Springer-Verlag 2011

Moshe Rappoport (IBM Research) trägt innerhalb des Unternehmens den interessanten Titel ,Executive Technology Briefer". Als solcher beschäftigt er sich mit dem Einfluss von IT-Trends auf die Geschäftsfelder von IBM. Er berichtete der Konzernspitze einmal jährlich und konzentriert sich in seinen Zukunftsberichten dabei auf verschiedene Zeitspannen (einige Jahre, fünf und zehn Jahre). Diese Berichte werden von ihm schon seit mehr als einem Jahrzehnt angefertigt, was ihm regelmäßig eine retrospektive Betrachtung erlaubt und ein Vergleich wie gut seine Vorhersagen den tatsächlichen Entwicklungen entsprachen. Und was soll man sagen? Die Jahresvorhersagen waren immer gut bis sehr gut. Kein Wunder, dauert es doch immer ein paar Jahre bis die Ideen aus den Forschungsabteilungen zur Marktreife gebracht werden können und da hat man als Wissenschaftler natürlich einen gewissen Vorsprung. Die Fünfjahresprognose war da schon weniger erfolgreich, wenngleich einige generelle Trends in dieser Zeitspanne durchaus prognostizierbar sind. Bei den Zehnjahresvorhersagen allerdings sah es häufig düster aus: wichtige technologische Entwicklungen nicht vorhergesehen oder auf die falschen gesetzt, entscheidende Marktveränderungen ignoriert oder nicht korrekt bewertet und äußere Rahmenbedingungen falsch gewichtet. Diese Liste an Fehleinschätzungen könnte beliebig weitergeführt werden und zeigt uns, dass längerfristige Zukunftsprognosen in den meisten Fällen keinen Sinn machen. Es ist mir nicht bekannt, ob IBM sich an diesen langfristigen Empfehlungen orientiert. Offensichtlich spielt es aber auch keine

\footnotetext{
A. Krüger $(\varangle)$

Deutsches Forschungszentrum für Künstliche Intelligenz und Universität des Saarlandes, Stuhlsatzenhausweg 3, 66123 Saarbrücken, Deutschland

e-mail: krueger@dfki.de
}

Rolle. Trotzdem ist der Wunsch weit nach vorne zu schauen sehr verständlich (und manchmal sowohl für Verfasser und Leser erhellend) und so werde auch ich versuchen als „Executive Technology Briefer“ des Fachbereichs „Künstliche Intelligenz" einen kleinen Zukunftsbericht zu unserem Fachbereich vorzulegen. Wie Moshe Rappoport möchte ich mich dabei an kurz-, mittel- und langfristigen Entwicklungen orientieren. Leider kann ich im Gegensatz zu meinem Kollegen von IBM keinerlei Aussagen über die Güte meiner Vorhersagen übernehmen, da ich mich das erste Mal an einer solchen versuche.

Anfang des Jahres habe ich das Amt des Sprechers von Uli Furbach übernommen und gemeinsam mit meinem designierten Stellvertreter Stefan Wölfl (Universität Freiburg) sind wir gerade dabei eine Bestandsaufnahme des Fachbereichs durchzuführen. Hierbei geht es vor allem darum zu erkennen, welche der auf unseren Webseiten gelisteten Fachgruppen und Arbeitskreise in welchem Maße aktiv sind, um gegebenenfalls Kräfte neu zu aktivieren oder zu bündeln. Die Webseiten werden im Anschluss der Bestandsaufnahme entsprechend angepasst. Erste Ergebnisse wollen wir dann breit im Fachbereich diskutieren. Wir prüfen zurzeit auch die Einrichtung eines KI-Literatur-Archivs in dem Schriften aus den Anfängen des Fachbereichs gesammelt und den Fachbereichsmitgliedern über unsere Webseite zur Verfügung gestellt werden können.

In den nächsten Jahren gibt es aus meiner Sicht eine Reihe von Herausforderungen zu meistern. Eine sehr wichtige Rolle wird die Stabilisierung unsere Mitgliederzahlen einnehmen. Zur Zeit hat der Fachbereich ca. 1300 Mitglieder und ist damit in den letzten fünf Jahren um ca. $20 \%$ geschrumpft. Während auch die GI selbst Mitglieder verliert und das Studienfach Informatik und damit auch die Künstliche Intelligenz in den letzten Jahren nicht von Studierenden überrannt wurden, muss uns das trotz allem zu den- 
ken geben. Der Fachbereich sollte vor allem für junge Mitglieder wieder attraktiver werden. Dies kann durch spezielle Förderideen für junge Nachwuchswissenschaftler geschehen oder durch Unterstützung von Studenten bei Tagungen und Workshops. Sicherlich könnten und sollten wir unser bestehendes Engagement in Schulen ausweiten und besser bekannt geben.

Die laufende Bestandsaufnahme wird sich auch auf die Wünsche und Bedürfnisse der Fachbereichsmitglieder, die nicht an Universitäten arbeiten, konzentrieren. Mein Eindruck ist, dass viele Unternehmen Methoden der KI verwenden, dies aber so nicht wahrnehmen. Dies zu ändern sollte ein weiteres Ziel für die nächsten Jahre sein. Die KIZeitschrift ist von meinen Vorgängern und den Herausgebern auf einen sehr guten Weg gebracht worden (darüber wird ja an anderer Stelle in diesem Heft berichtet). Diesen Weg wollen wir fortschreiten, die Themenvielfalt der Zeitschrift weiter erhalten und ausbauen. Für unseren Fachbereich von großer Bedeutung ist die jährlich stattfindende KITagung, die dieses Jahr in Berlin parallel zur Jahrestagung GI stattgefunden hat. Im Kanon der großen KI-Konferenzen geht sie manchmal ein wenig unter und spielt doch aus meiner Sicht eine wichtige Rolle. Denn durch die KI-Tagung konstituiert sich unsere Gemeinschaft. Sie dient dem Kennenlernen und dem breiteren Verständnis der KI-Landschaft, die durch unseren Fachbereich vertreten wird. Diese Funktion der KI-Tagung sollte aus meiner Sicht unbedingt in den nächsten Jahren weiter gestärkt werden. In Zeiten einer fast schon inflationären Menge an Tagungen, kann und sollte die KI-Tagung nicht als reines Veröffentlichungsorgan dienen, sondern vielmehr unsere Gemeinschaft stärken und das Verständnis füreinander fördern.

Wir werden uns bemühen, die Gründung neuer Fachgruppen und Arbeitskreise, die sich neuen für die KI relevanten Themenfeldern widmen, zu unterstützen. Welche Themen dies sein könnten wird sich zeigen. Das Ubiquitous Computing birgt sicherlich viele Chancen und neue Betätigungsfelder für unseren Fachbereich, ebenso wie die Robotik. Ähnlich wie in der Informatik halte ich auch in der KI eine Spezialisierung auf Anwendungsfelder für möglich und sinnvoll, ganz analog zur Entwicklung in der Medien, Geo-, und Bioinformatik. Die Künstliche Intelligenz sollte sich aus meiner Sicht auch vermehrt gesellschaftlich relevanten Themenfeldern zuwenden. Sei es zur Schonung von Ressourcen oder ihrem effizienten Einsatz, z.B. bei der Energiegewinnung oder der Wasserversorgung. Große Bedeutung werden zukünftig in unserem Alltag intelligente Assistenzsystem gewinnen. Dabei spielt es keine Rolle, ob diese im Auto, im Haushaltsroboter oder in der Rechenwolke ihre Benutzer begleiten. Aus meiner Sicht bezeichnet dies auch einen wichtigen Trend, der dazu beitragen kann die Attraktivität unseres Faches beim Nachwuchs zu steigern: der Fokus auf die menschenzentrierte Künstliche Intelligenz, wie sie sich teilweise schon in den Kognitionswissenschaften widerspiegelt und ebenso in vielen innovativen und intelligenten Benutzerschnittstellen, die an unseren Universitäten und in einigen Firmen erforscht werden mit vielen Berührungspunkten zu den Sozialwissenschaften. Die Verknüpfung von Mensch-Maschine Kommunikation, Ergonomie, einer benutzer-zentrierten Entwicklung und Methoden der KI sind der Leim aus denen große Teile zukünftiger Softwaresysteme bestehen werden. Dies deutlicher herauszuarbeiten wird eine mittelfristige Aufgabe für unseren Fachbereich sein.

Schließlich noch der Blick in die ferne Zukunft. Was soll man einer Verwaltungseinheit für die ferne Zukunft am Besten wünschen? Als Mitglied einer größeren Forschungseinrichtung und einer deutschen Universität mit entsprechender Verwaltungserfahrung rutscht einem da schnell ein „dass sie sich überflüssig macht!" heraus. Auch wenn etwas drastisch formuliert, wäre vielleicht genau dies das Wunschziel: die $\mathrm{KI}$ als allgegenwärtiger Bestandteil der Informatik in Ausbildung und der beruflichen Praxis. So stark in diese verankert, dass ein spezieller Fachbereich gar nicht mehr notwendig ist, da die von ihm geförderten Inhalte einer Förderung gar nicht mehr bedürfen. Ich würde mich freuen, wenn wir gemeinsam auf dieses Ziel zuarbeiten könnten und freue mich schon auf die nächsten Jahre der Zusammenarbeit im Fachbereich „Künstliche Intelligenz“. 